\title{
Re-definition of Blanking Period in Radiofrequency Catheter Ablation of Atrial Fibrillation in the Contact Force Era
}

\author{
Shunsuke Uetake ${ }^{1}$, Yasushi Miyauchi ${ }^{2}$, Tatsuya Mitsuishi ${ }^{1}$, Mitsunori Maruyama ${ }^{3}$, Seino \\ Yoshihiko $^{1}$, and Wataru Shimizu ${ }^{4}$ \\ ${ }^{1}$ Nippon Medical School Chiba Hokusoh Hospital \\ ${ }^{2}$ Nippon Medical School Chiba-Hokusoh Hospital \\ ${ }^{3}$ Nippon Medical School Musashi-Kosugi Hospital \\ ${ }^{4}$ Nippon Medical School
}

May 6, 2020

\begin{abstract}
Introduction: Early recurrence (ER) of atrial fibrillation (AF) is defined as the recurrence of atrial tachyarrhythmias within 3 months after AF ablation, however, this definition is based on data from the era of radiofrequency catheter ablation (RFCA), without contact force $(\mathrm{CF})$ technology. We investigated the significance of ER as a risk factor for late recurrence (LR) in paroxysmal AF (PAF) patients treated with CF and non-CF-guided ablation. Methods and Results: We studied 395 patients with PAF who underwent RFCA. Of these, 97 patients underwent RFCA without CF technology (Non-CF group) and 298 underwent with CF technology (CF group). Over a 2-year post-ablation follow-up period, LR occurred in 54 of 97 (55.7\%) patients in the Non-CF group, and in 105 of 298 (35.2\%) patients in the CF group. ER had a more significant relationship with LR in the CF than in the Non-CF group, and all patients in the CF group with ER in the 3rd month developed LR. Conclusion: ER in PAF patients who have undergone CF-guided ablation have a greater risk of LR than those who have undergone nonCF-guided ablation. ER in the 3rd month after CF-guided ablation may indicate an absolute risk of LR. Blanking period could be defined as 2 months in the CF era.
\end{abstract}

\section{Re-definition of Blanking Period in Radiofrequency Catheter Ablation of Atrial Fibrillation in the Contact Force Era}

Shunsuke Uetake, $\mathrm{MD}, \mathrm{PhD}^{1}$, Yasushi Miyauchi, $\mathrm{MD}, \mathrm{PhD}^{1}$, Tatsuya Mitsuishi, $\mathrm{MD}^{1}$, Mitsunori Maruyama, $\mathrm{MD}, \mathrm{PhD}^{2}$, Yoshihiko Seino, $\mathrm{MD}, \mathrm{PhD}^{1}$, Wataru Shimizu, $\mathrm{MD}, \mathrm{PhD}^{3}$

\section{Institution of authors:}

${ }^{1}$ Department of Cardiovascular Medicine, Nippon Medical School Chiba-Hokusoh Hospital

${ }^{2}$ Department of Cardiovascular Medicine, Nippon Medical School Musashi-Kosugi Hospital

${ }^{3}$ Department of Cardiovascular Medicine, Nippon Medical School

Conflicts of all authors: none declared.

Funding sources: none.

\section{Corresponding author}

Shunsuke Uetake, MD, PhD

Department of Cardiovascular Medicine, Nippon Medical School Chiba Hokusoh Hospital 
1715 Kamagari, Inzai, Chiba, 270-1694, Japan

E-mail address: s00-015@nms.ac.jp

\begin{abstract}
Introduction: Early recurrence (ER) of atrial fibrillation $(\mathrm{AF})$ is defined as the recurrence of atrial tachyarrhythmias within 3 months after AF ablation, however, this definition is based on data from the era of radiofrequency catheter ablation (RFCA), without contact force (CF) technology. We investigated the significance of ER as a risk factor for late recurrence (LR) in paroxysmal AF (PAF) patients treated with $\mathrm{CF}$ and non-CF-guided ablation.

Methods and Results : We studied 395 patients with PAF who underwent RFCA. Of these, 97 patients underwent RFCA without CF technology (Non-CF group) and 298 underwent with CF technology (CF group). Over a 2-year post-ablation follow-up period, LR occurred in 54 of 97 (55.7\%) patients in the NonCF group, and in 105 of $298(35.2 \%)$ patients in the CF group. ER had a more significant relationship with LR in the CF than in the Non-CF group, and all patients in the CF group with ER in the $3^{\text {rd }}$ month developed LR.
\end{abstract}

Conclusion : ER in PAF patients who have undergone CF-guided ablation have a greater risk of LR than those who have undergone non-CF-guided ablation. ER in the $3^{\text {rd }}$ month after CF-guided ablation may indicate an absolute risk of LR. Blanking period could be defined as 2 months in the CF era.

Key Words : Atrial fibrillation, contact force, early recurrence, blanking period, ablation

\title{
INTRODUCTION
}

Electrical isolation of the pulmonary veins (PVI) is a radical treatment for paroxysmal atrial fibrillation (PAF). Despite the acute success of PVI, recurrence of atrial tachyarrhythmia is common. Recurrence of atrial tachyarrhythmias within the first 3 months after an atrial fibrillation (AF) ablation procedure is defined as early recurrence (ER). ER is distinguished from late recurrence (LR) of atrial tachyarrhythmia, because only half of patients with ER also have LR. ${ }^{1}$ Accordingly, guidelines and expert consensus statements do not recommend early re-ablation during the first 3 months. However, some studies have shown that ER occurring in a later period was more strongly associated with long-term ablation failure in patients who underwent PVI using local electrode attenuation and impedance drop-guided radiofrequency catheter ablation (RFCA) and more than $90 \%$ of patients with ER during the $3^{\text {rd }}$ month post-ablation develop LR. ${ }^{2,3}$

Recently, the conventional ablation procedure has been replaced by contact force (CF) technology for PVI, and some studies have shown that CF-guided PVI is more effective in reducing AF recurrence than the conventional non-CF-guided PVI. ${ }^{4}$ However, the significance of ER episodes after CF-guided ablation in terms of LR remains unclear. Therefore, the objective of this study was to compare the significance of ER as a risk factor for LR in patients with paroxysmal AF (PAF) treated by CF and non-CF-guided ablation procedures.

\section{METHODS}

\section{Study Population}

This study included 395 consecutive patients who underwent their first RFCA procedure for PAF between February 2012 and June 2019 at Nippon Medical School Chiba-Hokusoh Hospital. This study was approved by the institutional review board. Patients were excluded from the study if any of the following were present: (1) severe valvular disease, (2) left ventricular ejection fraction $<35 \%$, (3) left atrial dimension $>55 \mathrm{~mm}$, (4) active thyroid disease, (5) hypertrophic cardiomyopathy, (6) hemodialysis, or (7) use of antiarrhythmic drugs during the blanking period. Ninety-seven patients underwent conventional non-CF-guided ablation (NonCF group) and 298 patients underwent CF-guided ablation (CF group: force-time integral [FTI]-guided, 181 patients; ablation index [AI]-guided, 117 patients).

\section{Ablation Procedure}


We performed all RFCA procedures under deep sedation. A duo-decapolar electrode catheter (BeeAT, Japan Lifeline, Tokyo, Japan) was inserted into the coronary sinus (CS) from the right internal jugular vein. Two long sheaths (Swarts and Agilis, Abbott, St. Paul, MN) were advanced into the left atrium through a single transseptal puncture via the right femoral vein. A duo-decapolar ring catheter (Lasso, Biosense Webster, Irvine, CA) was used to record pulmonary vein (PV) potential. Patients were anticoagulated with intravenous heparin to maintain an activated clotting time $>300$ seconds. All patients underwent circumferential PVI by isolating the left and right PVs as ipsilateral common ostia on the 3D-geometry of the left atrium (Carto 3, Biosense Webster). In patients with a long superior vena cava (SVC) sleeve (> 30 $\mathrm{mm}$ ) or in whom ectopic beats originating from the SVC triggered AF, electrical isolation of the SVC was performed as previously described. ${ }^{5}$ Isolation of the posterior wall of the left atrium (PLA) was performed depending on the operators' discretion. ${ }^{6}$

In the Non-CF group, ablation was performed with a 3.5-mm tip, 56-hole irrigated catheter (THERMOCOOL SF; Biosense Webster). In the CF group, a 3.5-mm tip, 56-hole irrigated catheter with a CF sensor (THERMOCOOL SMARTTOUCH SF, Biosense Webster) was used for ablation. Power settings were 25$30 \mathrm{~W}$ for the posterior wall and $30-35 \mathrm{~W}$ for other regions of the left atrium. In the Non-CF group, the RF current was delivered for 20-40 seconds to each lesion, until local bipolar electrograms decreased. If intra-esophageal temperature rose $>39.5$ during ablation, RF delivery was interrupted.

\section{CF-Guided Ablation}

In the CF group, FTI or AI targets were used, depending on the time period of the ablation procedure. Real-time automated display of RF applications (Visitag@, Biosense Webster) was used with predefined settings of catheter stability ( $1.5 \mathrm{~mm}$ for 3 seconds) and minimum CF ( $25 \%$ of the time $>3$ gram). Each inter-lesion distance was less than $6 \mathrm{~mm}$. In patients who underwent FTI-guided ablation, operators followed the EFFICAS I-based CF guidelines (minimum 400 gs FTI). ${ }^{7}$ In AI-guided ablation, RF was delivered until an AI of [?] 400 at the posterior wall and roof/bottom and [?] 550 at the anterior wall was achieved, in accordance with the CLOSE protocol. ${ }^{8}$

\section{RF Delivery Time and Delivered RF Energy}

RF delivery time was defined as the total time of RF application during PVI and, the delivered RF energy was defined as the total amount of RF energy delivered during PVI.

\section{Biomarkers of Inflammation}

Blood samples were taken to determine white blood cell count (WBC) and C-reactive protein (CRP) level before and at 1 day after the ablation procedure. We evaluated the ratio of WBC and the difference in CRP level between these two time-points.

\section{Postablation Follow-Up}

After the ablation procedures, all patients were discharged without antiarrhythmic drugs, and were followed up at $1,3,6,9$, and 12 months and then every 6 months thereafter until 24 months. At each hospital visit, patient symptoms and a 12-lead ECG were recorded. If patients reported symptoms, we asked them to undergo ECG with a portable electrocardiographic monitor (HCG-901; OMRON, Kyoto, Japan) at least twice a day and at any time they experienced palpitations during the follow-up period. Twenty-four-hour Holter ECG was performed at 1, 3, 6, 12, 18, and 24 months post-procedure. Recurrence of atrial tachyarrhythmia was defined as any documented atrial tachyarrhythmia (AF or atrial tachycardia [AT]) lasting $>30$ seconds on either device. ER was defined as any atrial tachyarrhythmia occurring during the first 3 months post-AF ablation and LR was defined as atrial tachyarrhythmias occurring after the first 3 months post-AF ablation.

\section{Statistical Analysis}

Continuous variables are presented as the mean +- SD and categorical variables as the number and percentage of patients. Two-group comparisons were performed with either Student's $t$-test or the Mann-Whitney U test according to the results of the Shapiro-Wilk normality test. Categorical variables were compared using 
Fisher's exact test. The cumulative atrial tachyarrhythmia recurrence-free survival was evaluated using the Kaplan-Meier method, and curves were compared using the log-rank test. Hazard ratios were used to determine the impact of the timing of the last ER on long-term atrial tachyarrhythmia recurrence and were calculated using a Cox proportional hazards model.

A p value $<0.05$ was considered statistically significant. When comparing three groups, Bonferroni correction was used, and a $\mathrm{p}$ value $<0.017$ was considered significant. Analyses were performed using IBM SPSS Statistics software version 21, (IBM Corporation, Armonk, NY).

\section{RESULTS}

\section{Patient Characteristics}

Patients baseline characteristics are compared between the two groups in Table 1. In the CF group, age and the $\mathrm{CHA}_{2} \mathrm{DS}_{2}$-VASc score were significantly higher, and disease duration of PAF was significantly longer than in the Non-CF group. The other clinical characteristics and echocardiographic data were comparable between the two groups.

\section{Results of Ablation}

Acute PVI, verified by entrance and exit blocks, was achieved in all patients. In the Non-CF group, additional SVC or PLA isolation were performed in 24 patients $(24.7 \%)$, while, in the CF group, additional SVC or PLA isolation were performed in 102 patients (34.2\%).

Parameters associated with ablation are shown in Table 2. The CF group had a shorter total RF delivery time and smaller amount of RF energy delivered than the Non-CF group. Regarding the post-ablation inflammatory response, the ratio of pre- to post-ablation WBC and the elevation of CRP levels were significantly lower in the CF group than in the Non-CF group.

\section{Incidence and Characteristics of Recurrence}

ER occurred in 159 patients overall (40.3\%). The incidence of ER was significantly greater in the Non-CF group than in the CF group (Table $2,55.7 \%$ vs. $35.2 \%$; $<<0.001$ ). In the CF group, the incidence of ER was significantly lower in patients in whom AI- $(18.8 \%)$ than in whom FTI-guidance $(42.5 \%)$ was used.

LR occurred in 42 patients (43.3\%) in the Non-CF group during an average follow-up period of 14.8 months after a single procedure, and in 65 patients $(21.8 \%)$ in the $\mathrm{CF}$ group during an average follow-up period of 12.4 months. The incidence of LR was significantly greater in Non-CF group than in the CF group (Figure 1, Table 2).

\section{Clinical Characteristics of Patients with Early or Late Recurrence}

In the Non-CF group, there was no significant difference in the baseline patient characteristics, RF delivery time, and delivered RF energy between the patients with and without ER (data not shown). On the other hand in the CF group, the patients with ER included a higher proportion of females, had a longer disease duration of PAF, higher pre-operative plasma brain-type natriuretic peptide (BNP) level, longer RF delivery time, and larger amount of RF delivery than those without ER (Female, $42.9 \%$ vs. $24.9 \%$, $\mathrm{p}=0.001$; disease duration of PAF, $41.2+-41.4$ vs. $25.5+-24.4$ months, p<0.001; BNP, $66.9+-58.7$ vs. $43.5+-33.1 \mathrm{pg} / \mathrm{ml}$, $\mathrm{p}<0.001$; RF delivery time, $2185.4+-585.2$ vs. $1963.4+-476.1$ sec., $\mathrm{p}=0.017$; amount of RF energy, 56349.7 +- 14616.6 vs. $52239.3+-12397.5 \mathrm{~J}, \mathrm{p}=0.070)$.

In the Non-CF group, the baseline patient characteristics was not different between those with and those without LR (data not shown). In the CF group, the disease duration of PAF was longer in patients with LR than in those without LR $(37.4+-34.6$ vs. $30.3+-34.6$ months, $\mathrm{p}=0.005)$, and the prevalence of hypertension, the $\mathrm{CHA}_{2} \mathrm{DS}_{2}$-VASc score, and pre-procedural BNP level were higher in those with than in those without LR(Hypertension, $66.2 \%$ vs. $49.8 \%, \mathrm{p}=0.013$; $\mathrm{CHA}_{2}$ DS2-VASc score, $2.2+-1.2$ vs. 1.8 +$1.2, \mathrm{p}=0.042$; BNP, $62.2+-51.2$ vs. $50.3+-45.2 \mathrm{pg} / \mathrm{ml}, \mathrm{p}=0.026)$. 


\section{Timing of Early Recurrence}

The timing of ER is summarized in Figure 2. In each post-ablation month, the incidence of ER was significantly greater in the Non-CF group. ER occurred in multiple months in $35.1 \%$ and in $9.1 \%$ of the patients in the Non-CF and CF group, respectively $(\mathrm{p}<0.001)$. The last ER most frequently occurred in the $3^{\text {rd }}$ month in the Non-CF group but in the $1^{\text {st }}$ month in the CF-group (Figure 3 ).

\section{Relationship between Early and Late Recurrence}

In the Non-CF group, 32 of 54 patients (59.3\%) with ER developed LR. The proportion of patients with ER was significantly greater among patients with LR than among those without LR $(76.2 \%$ vs. $40.0 \%$, p $<0.001)$. There was no difference in the rate of a single ER between patients with and without LR $(23.3 \%$ vs. $18.5 \%, \mathrm{p}=0.373)$, whereas the proportion of patients with ER occurring in multiple months were significantly greater in patients with LR than in those without LR $(51.2 \%$ vs. $22.2 \% \mathrm{p}=0.001)$. Freedom from LR in patients with the last ER in the $1^{\text {st }}$ or $2^{\text {nd }}$ month was significantly greater than in those with the last ER in the $3^{\text {rd }}$ month $(\mathrm{p}<0.001$; Figure $4 \mathrm{a})$.

Similarly, in the CF group, patients with LR had a higher rate of ER than those without LR (84.6\% vs. $21.5 \%, \mathrm{p}<0.001)$. However, in the CF group, the proportions of patients with ER in both single month and multiple months were significantly greater in patients with LR than in those without LR (single ER: $47.7 \%$ vs. $20.2 \%, \mathrm{p}<0.001$; multiple ER: $36.9 \%$ vs. $1.3 \%, \mathrm{p}<0.001$ ). The rate of freedom from LR in patients with the last ER occurring in the $1^{\text {st }}$ and $2^{\text {nd }}$ months was $69.8 \%$ and $40.0 \%$, respectively, whereas all patients with the last ER occurring in the $3^{\text {rd }}$ month had LR (Figure $4 \mathrm{~b}$ ).

When comparing patients with the last ER occurring in the $3^{\text {rd }}$ month in the CF and Non-CF groups, the CF group was significantly less likely to be free from LR at 2 years post-procedure than the Non-CF group (Figure 4c). The significance of ER as a risk factor of LR is summarized in Table 3. Occurrence of the last ER in the $1^{\text {st }}$ or $2^{\text {nd }}$ month was not a significant risk factor for LR in the Non-CF group, whereas any ER at any time-point was a significant risk factor for LR in the CF group. Of note, occurrence of the last ER in the $3^{\text {rd }}$ month was an absolute risk factor for LR in the CF group.

\section{Relationship between ER and PV Reconnection}

Among 157 patients with ER, 74 patients underwent repeat RFCA for LR (Non-CF: 32 [59.3\%]; FTI: 34 [44.2\%]; AI: 8 [28.6\%]). Patients without ER were excluded from the analysis. Reconnection of any PV was observed in $93.8 \%, 70.6 \%$, and $25 \%$ of patients in the Non-CF, FTI, and AI group, respectively (Figure 5). Patients who underwent AI-guided ablation were less likely to have PV reconnection than those who underwent FTI-guided ablation.

\section{DISCUSSION}

\section{Main Findings}

The main findings of this study were that patients who underwent CF-guided ablation less frequently developed ER than those who underwent non-CF-guided ablation. Moreover, all patients in the CF group with ER occurring in the $3^{\text {rd }}$ month developed LR, although $22.2 \%$ of patients in the Non-CF group who had ER in the $3^{\text {rd }}$ month were free-from LR during 2-year follow-up period. These findings suggest that ER occurring in the $3^{\text {rd }}$ month is a specific risk factor for LR and that an early redo-procedure may be reasonable in patients who underwent PVI using CF technology.

\section{Mechanism of Early Recurrence}

Previous studies with patients in the non-CF era have reported an approximately $50 \%$ prevalence of ER after ablation and a higher incidence of LR, ranging between $41.1 \%$ and $90 \%$, in cases with ER during the 3-month blanking period. ${ }^{3,}$ 9, 10 The present study showed that $55.7 \%$ patients in the Non-CF group had ER during the 3-month blanking period and $59.3 \%$ of them had LR. These results were consistent with those of 
previous studies. On the other hand, in CF ablation cases, only $35.2 \%$ cases experienced ER, and $52.4 \%$ of those with ER also had LR. These results were also consistent with those of a previous study. ${ }^{11}$

The mechanisms of ERs are considered to be post-ablation transient factors, such as inflammatory changes, in addition to reconnection of PV or residual extra-PV triggers. ${ }^{1}$ A previous histopathological study had demonstrated that RF energy delivery causes inflammatory responses after the ablation procedure.${ }^{12}$ Furthermore, a greater inflammatory response after an RF ablation procedure has been associated with ER, and a longer $\mathrm{RF}$ ablation time and total procedure time are significant predictors for post-ablation inflammation and myocardial injury. ${ }^{13}$ In the present study, the degree of elevation in WBC and CRP levels after ablation was significantly smaller in patients who underwent CF-guided ablation (Table 2). The RF delivery time was shorter, and the amount of RF energy delivered was smaller in patients who underwent CF-guided ablation (Table 2). Appropriate force of tissue-catheter contact, and stability of RF delivery provided by CF technology may result in more successful PVI, without excessive RF delivery, and will reduce the inflammatory response. A lower inflammatory response may partly explain the lower incidence of ER in the CF-group.

\section{Different Time Course of Early Recurrence and Significance for Late Recurrence}

A previous study has suggested that the incidence of ER decreases after 1-month post-ablation, in accordance with the normalization of the systemic inflammatory response as assessed by WBC and CRP levels. ${ }^{13}$ Histological examinations after RF delivery have demonstrated that myocardial tissue conversion to fibrotic and fatty tissue is not completed within 1 month, as macrophages and lymphocytes are still present in the ablation lesion for 4-8 weeks after ablation. ${ }^{14}$ Thus, local tissue inflammation may persist after 1 month. In the present study, the greatest number of patients experienced ER in the $1^{\text {st }}$ month after PAF ablation, and ER occurrence decreased in the $2^{\text {nd }}$ and even more in the $3^{\text {rd }}$ month, in both groups. A decrease in the inflammatory changes can explain the time-dependent decrease in the incidence of ER. However, the timing of the last ER for most patients in the Non-CF group was the $3^{\text {rd }}$ month, whereas it was the $1^{\text {st }}$ month in the $\mathrm{CF}$ group. Lower initial inflammatory changes in the $\mathrm{CF}$ group may result in earlier disappearance of inflammatory changes. Furthermore, $78 \%$ of patients in the Non-CF group with ER in the $3^{\text {rd }}$ month developed LR, whereas $100 \%$ of patients in the CF group with ER in the $3^{\text {rd }}$ month developed LR. Therefore, recurrence in the $3^{\text {rd }}$ month may be due to residual triggers, including reconnection of the PVI lesion, in the CF group. With CF technology, the blanking period can be defined as the first 2-month period, and patients with ER in the $3^{\text {rd }}$ month can be good candidates for an early redo-procedure. In addition, reconnection of the PV after the $2^{\text {nd }}$ procedure was less frequent in patients who undergo an AI-based ablation than in those who undergo an FTI-based ablation in the CF group, suggesting that AI-based ablation ensures more durable PVI than FTI-based or non-CF catheter-based PVI.

\section{Limitations}

This was a retrospective, single-center study with a limited number of cases. Multicenter randomized studies are necessary to verify the risk for LR in cases with ER in the $3^{\text {rd }}$ month. Modification of the autonomic nervous system (ANS) by RF ablation can be a transient cause of ER; however, we did not assess changes in the ANS in this study.

\section{Conclusions}

ER of atrial tachyarrhythmias in the $3^{\text {rd }}$ month after PAF ablation with CF technology was a specific indicator of LR. The blanking period can be defined as the first 2 months after ablation in patients undergoing PVI with CF technology, and an early $2^{\text {nd }}$ ablation procedure could be encouraged in patients who have ER in the $3^{\text {rd }}$ month.

\section{Acknowledgments: none.}

\section{REFERENCES}

1. Calkins H, Hindricks G, Cappato R, et al. 2017 HRS/EHRA/ECAS/APHRS/SOLAECE expert consensus statement on catheter and surgical ablation of atrial fibrillation.Heart Rhythm . 2017;14:e275-e444. 
2. Themistoclakis S, Schweikert RA, Saliba WI, et al. Clinical predictors and relationship between early and late atrial tachyarrhythmias after pulmonary vein antrum isolation. Heart Rhythm. 2008;5:679-685.

3. Willems S, Khairy P, Andrade JG, et al.; ADVICE Trial Investigators. Redefining the blanking period after catheter ablation for paroxysmal atrial fibrillation: Insights from the ADVICE (adenosine following pulmonary vein isolation to target dormant conduction elimination) trial. Circ Arrhythm Electrophysiol . 2016;9:e003909.

4. Jarman JWE, Panikker S, Das M, et al. Relationship between contact force sensing technology and medium-term outcome of atrial fibrillation ablation: a multicenter study of 600 patients. $J$ Cardiovasc Electrophysiol . 2015;26:378-384.

5. Higuchi K, Yamauchi Y, Hirao K, et al. Superior vena cava as initiator of atrial fibrillation: factors related to its arrhythmogenicity. Heart Rhythm.2010;7:1186-1191.

6. Sanders $\mathrm{P}$, Hocini $\mathrm{M}$, Jais $\mathrm{P}$, et al. Complete isolation of the pulmonary veins and posterior left atrium in chronic atrial fibrillation. Long-term clinical outcome.Eur Heart J . 2007;28:1862-1871.

7. Kautzner J, Neuzil P, Lambert H, et al. EFFICAS II: optimization of catheter contact force improves outcome of pulmonary vein isolation for paroxysmal atrial fibrillation. Europace . 2015;17:1229-1235.

8. Taghji P, El Haddad M, Phlips T, et al. Evaluation of a strategy aiming to enclose the pulmonary veins with contiguous and optimized radiofrequency lesions in paroxysmal atrial fibrillation: A pilot study. JACC Clin Electrophysiol . 2018;4:99-108.

9. Lellouche N, Jais P, Nault I, et al. Early recurrences after atrial fibrillation ablation: prognostic value and effect of early reablation. J Cardiovasc Electrophysiol . 2008;19:599-605.

10. Andrade JG, Khairy P, Verma A, et al. Early recurrence of atrial tachyarrhythmias following radiofrequency catheter ablation of atrial fibrillation. Pacing Clin Electrophysiol . 2012;35:106-116.

11. Miyazaki S, Kuroi A, Hachiya H, et al. Early recurrence after pulmonary vein isolation of paroxysmal atrial fibrillation with different ablation technologies - Prospective comparison of radiofrequency vs. secondgeneration cryoballoon ablation. Circulation J . 2016;80:346-353.

12. Grubman E, Pavri BB, Lyle S, Reynolds C, Denofrio D, Kocovic DZ. Histopathologic effects of radiofrequency catheter ablation in previously infarcted human myocardium. J Cardiovasc Electrophysiol . 1999;10:336-342.

13. Lim HS, Schultz C, Dang J, et al. Time course of inflammation, myocardial injury, and prothrombotic response after radiofrequency catheter ablation for atrial fibrillation. Circ Arrhythm Electrophysiol . 2014;7:83-89.

14. Tanno K, Kobayashi Y, Kurano K, et al. Histopathology of canine hearts subjected to catheter ablation using radiofrequency energy. Jpn Circ J . 1994;58:123-135.

Table 1. Baseline patient characteristics

\begin{tabular}{llll}
\hline Characteristics & Non-CF $(\mathrm{n}=97)$ & $\mathrm{CF}(\mathrm{n}=298)$ & $\mathrm{p}$ value \\
\hline Age $($ year) & $63.7 \pm 9.7$ & $65.3 \pm 9.9$ & 0.085 \\
Female, $\mathrm{n}(\%)$ & $20(20.6 \%)$ & $93(31.2 \%)$ & 0.028 \\
BMI $\left(\mathrm{kg} / \mathrm{m}^{2}\right)$ & $24.0 \pm 3.1$ & $24.1 \pm 3.5$ & 0.485 \\
PAF Disease Duration (months) & $24.9 \pm 42.2$ & $32.1 \pm 33.5$ & 0.023 \\
HT, n $(\%)$ & $51(52.6 \%)$ & $159(53.4 \%)$ & 0.493 \\
CHF, n (\%) & $2(2.1 \%)$ & $14(4.7 \%)$ & 0.203 \\
DM, n (\%) & $13(13.4 \%)$ & $30(10.1 \%)$ & 0.230 \\
Previous stroke or TIA, n $(\%)$ & $1(1.0 \%)$ & $10(3.4 \%)$ & 0.202
\end{tabular}




\begin{tabular}{llll}
\hline Characteristics & Non-CF $(\mathrm{n}=97)$ & $\mathrm{CF}(\mathrm{n}=298)$ & $\mathrm{p}$ value \\
\hline Vascular disease, $\mathrm{n}(\%)$ & $1(1.0 \%)$ & $17(5.7 \%)$ & 0.055 \\
CHA $_{2} \mathrm{DS}_{2}$-VASc score & $1.51 \pm 1.13$ & $1.94 \pm 1.26$ & 0.010 \\
T-cho $(\mathrm{mg} / \mathrm{dl})$ & $199.3 \pm 34.5$ & $190.9 \pm 32.8$ & 0.523 \\
LDL-cho $(\mathrm{mg} / \mathrm{dl})$ & $114.4 \pm 29.9$ & $108.4 \pm 28.3$ & 0.958 \\
Hba1c $(\%)$ & $5.9 \pm 0.6$ & $5.9 \pm 0.7$ & 0.509 \\
BNP $(\mathrm{pg} / \mathrm{ml})$ & $45.2 \pm 43.0$ & $53.3 \pm 46.9$ & 0.081 \\
Ccr $(\mathrm{ml} / \mathrm{min})$ & $83.7 \pm 23.1$ & $77.4 \pm 27.7$ & 0.036 \\
Echocardiographic Data & & & \\
LAD $(\mathrm{mm})$ & $40.6 \pm 5.9$ & $41.0 \pm 6.0$ & 0.709 \\
LA area $\left(\times 10^{2} \mathrm{~mm}^{2}\right)$ & $20.2 \pm 5.4$ & $21.4 \pm 5.5$ & 0.035 \\
EF $(\%)$ & $65.5 \pm 8.4$ & $65.8 \pm 7.7$ & 0.863 \\
Catheter ablation procedure & & & \\
Additional SVC/PLA ablation & $24(24.7 \%)$ & $102(34.2 \%)$ & 0.082 \\
\hline
\end{tabular}

Continuous variables are presented as mean $\pm \mathrm{SD}$.

$\mathrm{AF}$, atrial fibrillation; BMI. body mass index; BNP, brain natriuretic peptide; Ccr, creatinine clearance; CF, contact force; $\mathrm{CHF}$, congestive heart failure; DM, diabetes mellitus; EF, left ventricular ejection fraction; HT, hypertension; LDL-cho; low-density lipoprotein cholesterol; LAD, left atrial dimension; LA, left atrial; PLA, posterior wall of the left atrium; SVC, superior vena cava; TIA, transient ischemic attack; T-cho, total cholesterol

Table 2. Difference of ablation results between the Non-CF and the CF group

\begin{tabular}{llll}
\hline Characteristics & Non-CF $(\mathrm{n}=97)$ & CF $(\mathrm{n}=298)$ & $\mathrm{p}$ value \\
\hline RF delivery time (sec) & $2401.1 \pm 733.4$ & $2056.8 \pm 534.5$ & $<0.001$ \\
delivered RF energy $(\mathrm{J})$ & $59130.9 \pm 19636.4$ & $53698.4 \pm 13487.2$ & 0.031 \\
WBC ratio & $1.72 \pm 0.33$ & $1.53 \pm 0.34$ & $<0.001$ \\
Difference of CRP $(\mathrm{mg} / \mathrm{dl})$ & $1.21 \pm 1.81$ & $0.73 \pm 0.93$ & 0.001 \\
Early Recurrence & $54(55.7 \%)$ & $105(35.2 \%)$ & $<0.001$ \\
AF & $44(45.4 \%)$ & $87(29.2 \%)$ & 0.003 \\
AT & $9(9.3 \%)$ & $12(4.0 \%)$ & 0.046 \\
Late Recurrence & $42(43.3 \%)$ & $65(21.8 \%)$ & $<0.001$ \\
AF & $31(32.0 \%)$ & $63(21.1 \%)$ & 0.023 \\
AT & $16(16.5 \%)$ & $5(1.7 \%)$ & $<0.001$ \\
\hline
\end{tabular}

Continuous variables are presented as mean $\pm \mathrm{SD}$.

$\mathrm{AF}$, atrial fibrillation; AT, atrial tachycardia; CF, contact force; CRP, C-reactive protein; LR, late recurrence; $\mathrm{RF}$, radiofrequency; $\mathrm{WBC}$, white blood cell count; $\mathrm{WBC}$ ratio $=$ post-ablation $\mathrm{WBC} /$ pre-ablation $\mathrm{WBC}$, Difference of CRP $=$ post-ablation CRP - pre-ablation CRP

Table 3. Characteristics and significance of early recurrence as the risk factor for late recurrence

\begin{tabular}{lllllll}
\hline & Non-CF $(\mathrm{n}$ & $\begin{array}{l}\text { Non-CF }(\mathrm{n} \\
=97)\end{array}$ & $\begin{array}{l}\text { Non-CF }(\mathrm{n} \\
=97)\end{array}$ & $\begin{array}{l}\text { CF }(\mathrm{n}= \\
298)\end{array}$ & $\begin{array}{l}\text { CF }(\mathrm{n}= \\
298)\end{array}$ & $\begin{array}{l}\text { CF }(\mathrm{n}= \\
298)\end{array}$ \\
\hline $\begin{array}{l}\text { ER } \\
\text { Characteristics }\end{array}$ & Late & OR $(95 \%$ CI $)$ & p Value & Late & OR (95\% CI) & p Value \\
& Recurrence & & & Recurrence & &
\end{tabular}




\begin{tabular}{|c|c|c|c|c|c|c|}
\hline & $\begin{array}{l}\text { Non-CF (n } \\
=97)\end{array}$ & $\begin{array}{l}\text { Non-CF (n } \\
=97)\end{array}$ & $\begin{array}{l}\text { Non-CF (n } \\
=97)\end{array}$ & $\begin{array}{l}\text { CF }(n= \\
298)\end{array}$ & $\begin{array}{l}\text { CF }(n= \\
298)\end{array}$ & $\begin{array}{l}\text { CF }(n= \\
298)\end{array}$ \\
\hline Any ER & $32(59.3 \%)$ & $\begin{array}{l}4.800 \\
(1.968-11.709)\end{array}$ & 0.001 & $55(52.4 \%)$ & $\begin{array}{l}20.130 \\
(9.577-42.311)\end{array}$ & $<0.001$ \\
\hline Single ER & $10(50.0 \%)$ & $\begin{array}{l}3.300 \\
(1.070-10.179)\end{array}$ & 0.038 & $31(39.7 \%)$ & $\begin{array}{l}8.657 \\
(4.192-17.879)\end{array}$ & $<0.001$ \\
\hline Multiple ERs & $22(64.7 \%)$ & $\begin{array}{l}6.050 \\
(2.231-16.407)\end{array}$ & $<0.001$ & $24(88.9 \%)$ & $\begin{array}{l}107.051 \\
(28.487- \\
402.294)\end{array}$ & $<0.001$ \\
\hline $1^{\text {st }}$ month only & $5(38.5 \%)$ & $\begin{array}{l}2.062 \\
(0.555-7.740)\end{array}$ & 0.283 & $19(30.2 \%)$ & $\begin{array}{l}5.676 \\
(2.599-12.395)\end{array}$ & $<0.001$ \\
\hline $\begin{array}{l}2^{\text {nd }} \text { month } \\
\text { only }\end{array}$ & $3(100 \%)$ & undefined & $<0.001$ & $4(57.1 \%)$ & $\begin{array}{l}17.128 \\
(3.459-84.819)\end{array}$ & $<0.001$ \\
\hline $3^{\text {rd }}$ month only & $2(50.0 \%)$ & $\begin{array}{l}3.300 \\
(0.411-26.514)\end{array}$ & 0.261 & $8(100 \%)$ & Undefined & $<0.001$ \\
\hline $\begin{array}{l}1^{\text {st }}+2^{\text {nd }} \\
\text { month }\end{array}$ & $3(27.3 \%)$ & $\begin{array}{l}1.237 \\
(0.275-5.567)\end{array}$ & 0.781 & $5(62.5 \%)$ & $\begin{array}{l}21.410 \\
(4.596-99.730)\end{array}$ & $<0.001$ \\
\hline $\begin{array}{l}1^{\text {st }}+3^{\text {rd }} \\
\text { month }\end{array}$ & $5(71.4 \%)$ & $\begin{array}{l}8.250 \\
(1.383-49.213)\end{array}$ & 0.021 & $4(100 \%)$ & Undefined & $<0.001$ \\
\hline $\begin{array}{l}2^{\text {nd }}+3^{\text {rd }} \\
\text { month }\end{array}$ & $4(100 \%)$ & undefined & $<0.001$ & $4(100 \%)$ & Undefined & $<0.001$ \\
\hline $\begin{array}{l}1^{\text {st }}+2^{\text {nd }}+3^{\text {rd }} \\
\text { month }\end{array}$ & $10(83.3 \%)$ & $\begin{array}{l}16.500 \\
(3.091-88.085)\end{array}$ & 0.001 & $11(100 \%)$ & Undefined & $<0.001$ \\
\hline $\begin{array}{l}\text { Last ER in } 1^{\text {st }} \\
\text { month }\end{array}$ & $5(38.5 \%)$ & $\begin{array}{l}2.062 \\
(0.555-7.740)\end{array}$ & 0.283 & $19(30.2 \%)$ & $\begin{array}{l}5.676 \\
(2.599-12.395)\end{array}$ & $<0.001$ \\
\hline $\begin{array}{l}\text { Last ER in } 2^{\text {nd }} \\
\text { month }\end{array}$ & $6(42.9 \%)$ & $\begin{array}{l}1.833 \\
(0.499-6.742)\end{array}$ & 0.362 & $9(60.0 \%)$ & $\begin{array}{l}19.269 \\
(5.938-62.528)\end{array}$ & $<0.001$ \\
\hline $\begin{array}{l}\text { Last ER in } 3^{\text {rd }} \\
\text { month }\end{array}$ & $21(77.8 \%)$ & $\begin{array}{l}11.550 \\
(3.655-36.497)\end{array}$ & $<0.001$ & $27(100 \%)$ & Undefined & $<0.001$ \\
\hline
\end{tabular}

$\mathrm{CF}$, contact force; ER, early recurrence

Figure and Figure Legends 


\section{Figure 1}

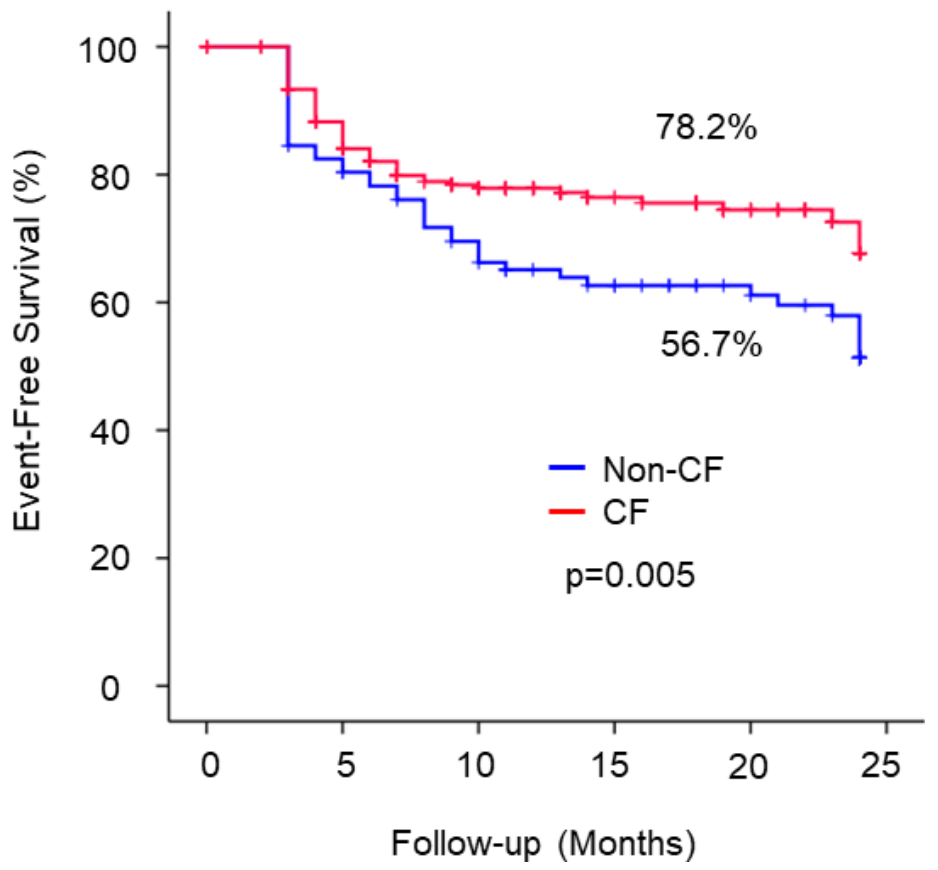

Figure 1. Freedom from atrial tachyarrhythmias in patients with paroxysmal atrial fibrillation after a single ablation procedure with or without contact force (CF) technology. Patients with CF-guided ablation were more likely to achieve long-term ablation success.

Figure 2. Incidence of early recurrence episodes in each month during the blanking period in patients with paroxysmal atrial fibrillation ablated with and without contact force $(\mathrm{CF})$ technology. In both groups, the incidence of early recurrence (ER) was highest in the $1^{\text {st }}$ month. In each month after ablation, the incidence of ER was higher in the non-CF group. 
Figure 2

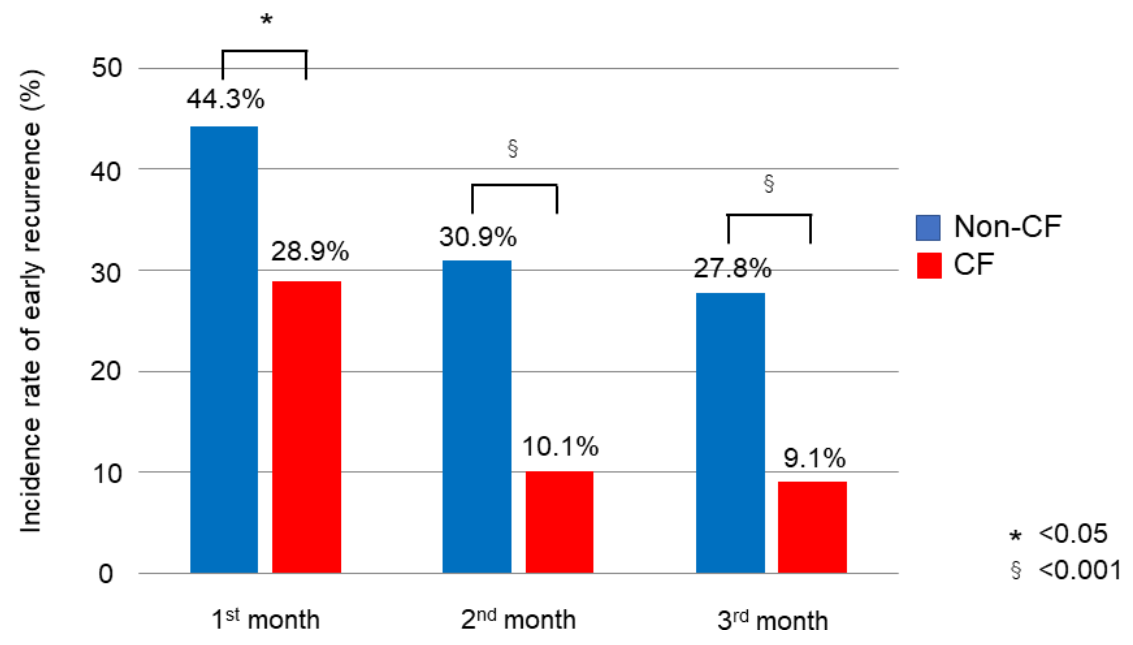

Figure 3. The timing of the last occurrence of early recurrence (ER) of atrial tachyarrhythmias in patients ablated with and without contact force (CF) technology. In the Non-CF group, the last ER episode occurred most frequently in the $3^{\text {rd }}$ month, whereas it occurred most frequently in the $1^{\text {st }}$ month in the CF group.

Figure 3

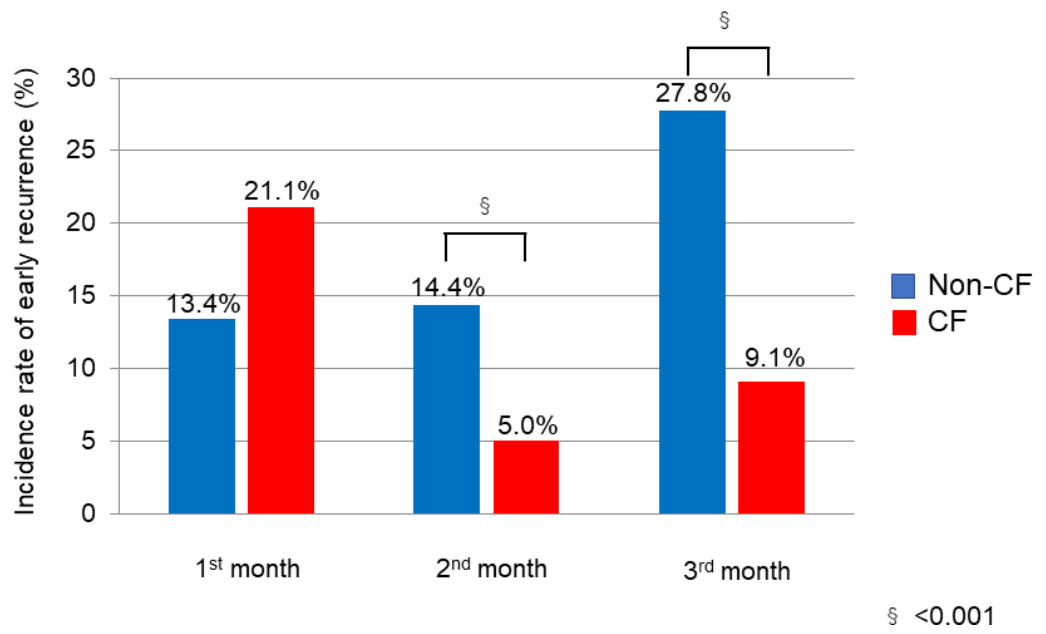




\section{Figure 4}

(c)

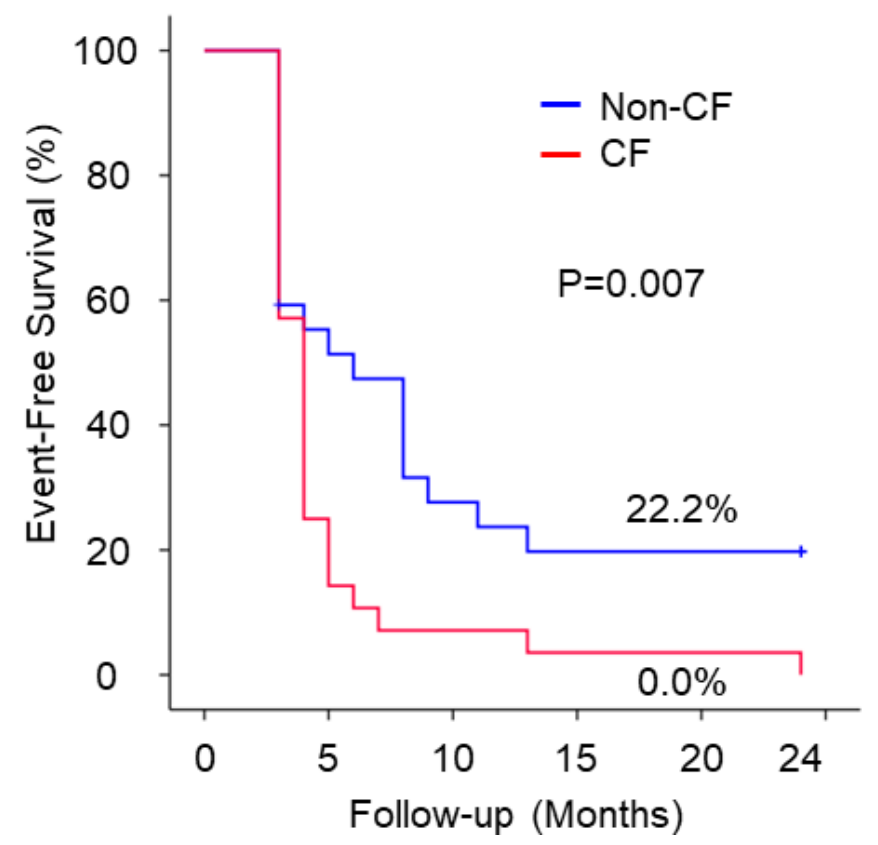

Figure 4

(a)

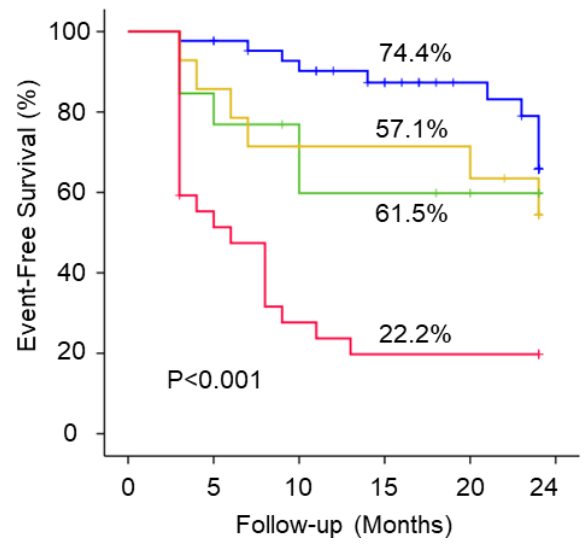

- No ER

- Last ER $1^{\text {st }}$ month

- Last ER $2^{\text {nd }}$ month

— Last ER $3^{\text {rd }}$ month

(b)

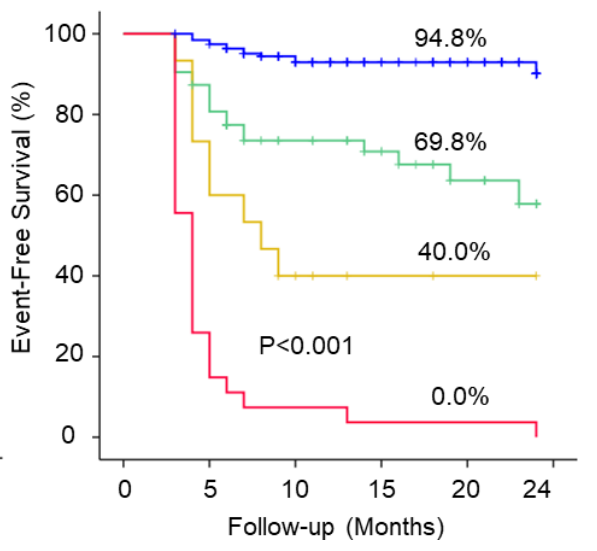

Figure 4. (a) Freedom from atrial tachyarrhythmia after the non-contact force-guided ablation procedure in 
patients with early recurrence (ER) in the $1^{\text {st }}, 2^{\text {nd }}$, and $3^{\text {rd }}$ month post-ablation. (b) Freedom from atrial tachyarrhythmia after a contact force $(\mathrm{CF})$-guided ablation procedure in patients with ER in the $1^{\text {st }}, 2^{\text {nd }}$, and $3^{\text {rd }}$ month post-ablation. (c) Comparison of atrial tachyarrhythmia-free survival in patients who had $\mathrm{ER}$ in the $3^{\text {rd }}$ month, in both $\mathrm{CF}$ and Non-CF groups.

Figure 5. Incidence of pulmonary vein (PV) reconnection in patients with paroxysmal atrial fibrillation with early recurrence (ER) after ablation, who underwent a redo-procedure. CF; contact force, FTI; force-time integral, AI; ablation index

Figure 5

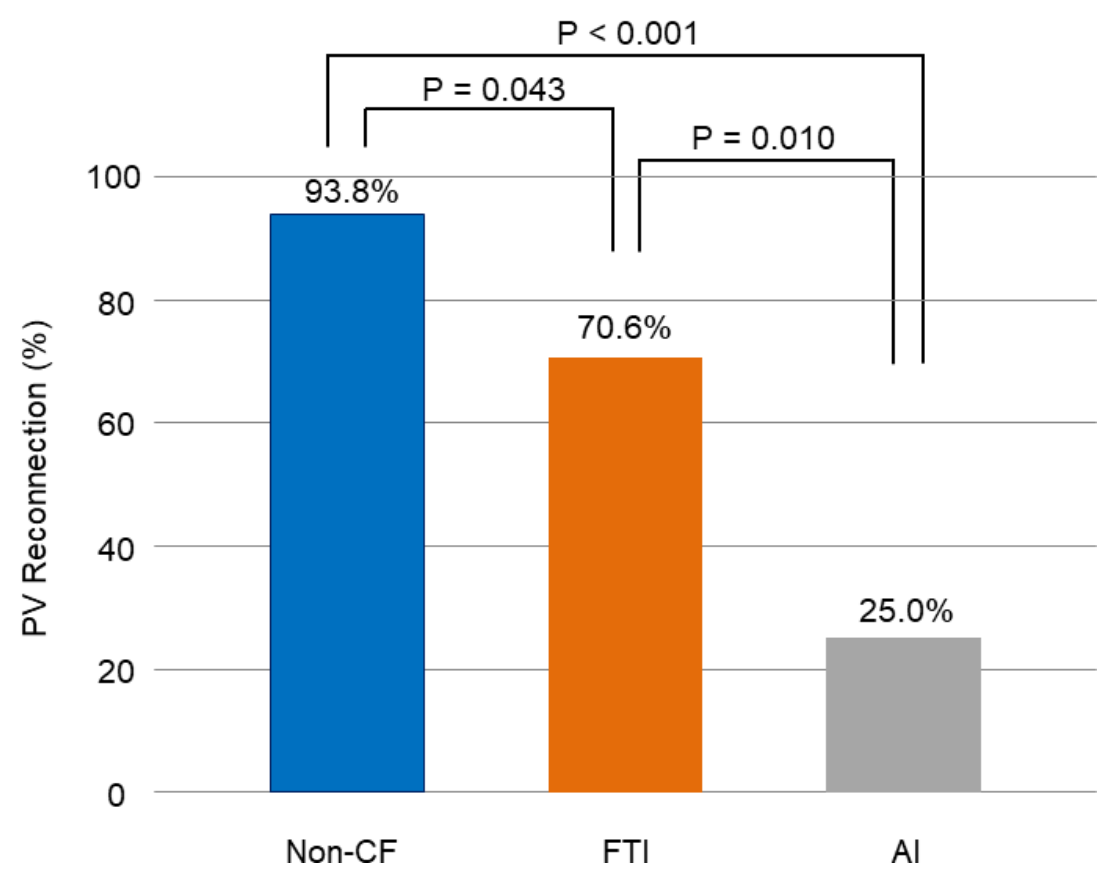

